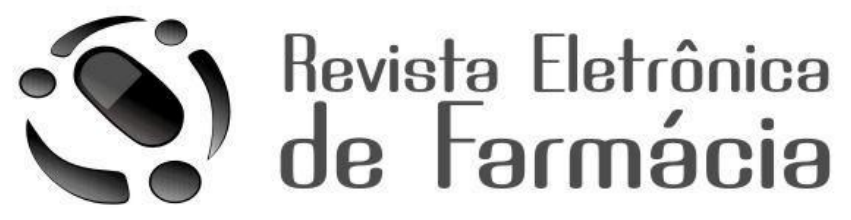

REF - ISSN 1808-0804 Vol. XI (1), 35 - 47, 2014.

\title{
REVISÃO INTEGRATIVA SOBRE O USO DE SISTEMAS INFORMATIZADOS NA PRÁTICA DA ATENÇÃO FARMACÊUTICA
}

\author{
INTEGRATIVE REVIEW ON THE USE OF COMPUTERIZED SYSTEMS IN \\ PHARMACEUTICAL CARE PRACTICE
}

REVISIÓN INTEGRATIVA SOBRE LA UTILIZACIÓN DE SISTEMAS INFORMÁTICOS EM LA PRATICA DE ATENCIÓN FARMACÉUTICA

\section{Carina da Costa Braúna ${ }^{1}$; Rivelilson Mendes de Freitas ${ }^{1, *}$}

${ }^{1}$ Curso de Farmácia da Universidade Federal do Piauí, Teresina, Piauí.

*e-mail para correspondência: rivelilson@pq.cnpq.br

Recebido em 01/06/2013, Aceito em 21/01/2014.

\begin{abstract}
RESUMO: O uso inadequado de medicamentos gera problemas relacionados com medicamentos (PRMs) tais como interações, reações adversas, entre outros. 0 acompanhamento farmacoterapêutico (AFT) ajuda a reduzir a probabilidade desses PRMs. Com a facilidade de acesso aos computadores, surgiram os sistemas informatizados como ferramentas úteis no bom monitoramento dos usuários de medicamentos. A presente investigação é uma revisão integrativa que teve como objetivo buscar e avaliar as evidências científicas disponíveis sobre o uso de sistemas informatizados na atenção farmacêutica nos últimos cinco anos. Para tanto, a seleção dos estudos foi realizada por meio de quatro bases de dados: PUBMED, MEDLINE, LILACS e SCIELO, resultando em 15 artigos científicos. A apresentação dos artigos incluídos nessa revisão integrativa foi distribuída em tabelas, considerando os seguintes pontos: título, autoria, objetivo e resultados/conclusão. Os resultados foram discutidos de forma descritiva e em consonância com o objetivo proposto. Destaca-se as vantagens da utilização desses softwares no acompanhamento farmacoterapêutico, devendo ser estimulada entre farmacêuticos. Contudo, torna-se necessário um maior conhecimento sobre a segurança dos mesmos para uma incorporação eficaz à prática clínica.
\end{abstract}

PALAVRAS-CHAVE: Farmacovigilância, Software, Tecnologia da Informação. 
FREITAS, M. R.

REF - ISSN 1808-0804 Vol. XI (1), 35 - 47, 2014.

\begin{abstract}
The inappropriate use of drugs creates problems related to drugs such as interactions, adverse reactions, among others. Pharmacotherapeutic follow-up helps reduce the likelihood of these problems. With the popularization of computers, computerized systems have emerged as useful tools in good pharmaceutical monitoring of drug users. This research is an integrative review aimed to search and evaluate the available scientific evidence on the use of computerized systems in pharmaceutical care in the last five years. Therefore, the selection of studies was performed using four databases: PubMed, MEDLINE, LILACS and SciELO, resulting in 15 scientific articles. The presentation of the articles included in this integrative review was distributed in tables considering the following points: title, author, purpose and results/conclusion. The results were discussed in a descriptive manner and in accordance with the proposed objective. We highlight the advantages of using such softwares in monitoring pharmacotherapy and therefore are encouraged among pharmacists. However it is necessary a better understanding of data security for an effective incorporation into clinical practice.
\end{abstract}

KEYWORDS: Pharmacovigilance, Software, Information Technology.

RESUMEN: El uso inadecuado de drogas genera problemas relacionados con las drogas (PRM), tales como interacciones, reacciones adversas, entre otros. El seguimiento farmacoterapéutico (AFT) ayuda a reducir la probabilidad de que estos PRM. Con la popularización de los ordenadores, sistemas informáticos se han convertido en herramientas útiles en el control de las drogas. La presente investigación es una revisión integradora tuvo como objetivo buscar y evaluar la evidencia científica disponible sobre el uso de sistemas informáticos en la atención farmacéutica en los últimos cinco años. Por lo tanto, la selección de los estudios se realizó mediante cuatro bases de datos: PubMed, MEDLINE, LILACS y SciELO, lo que resulta en 15 artículos científicos. La presentación de los artículos incluidos en esta revisión integradora se distribuyó en tablas, teniendo en cuenta los siguientes puntos: título, autoría, propósito y resultados / conclusión. Los resultados se analizaron de una manera descriptiva y de acuerdo con el objetivo propuesto. Destacamos las ventajas de utilizar este tipo de software en el seguimiento farmacoterapéutico, debe fomentarse entre los farmacéuticos. Sin embargo, es necesario un mayor conocimiento de su seguridad para una incorporación efectiva en la práctica clínica.

PALABRAS CLAVE: Farmacovigilancia, Software, Tecnologías de la Información.

\section{INTRODUÇÃO}

A Atenção Farmacêutica pode ser definida como a participação ativa do farmacêutico para assistência ao paciente na dispensação e seguimento de um tratamento farmacoterapêutico, cooperando assim com o médico e outros profissionais da saúde. Além disso, tem a finalidade de garantir uma farmacoterapia racional, segura e custo-efetiva ao paciente, obtendo resultados que melhorem a sua $v_{i d a}^{(1,2)}$.
É sabido que o uso inadequado de medicamentos, a administração de muitas medicações, a dose incorreta, o esquecimento da administração e a falta de padronização de horários geram problemas relacionados com medicamentos (PRMs) como interações, reações adversas, entre outros. O acompanhamento farmacoterapeutico (AFT) ajuda a reduzir a probabilidade desses PRMs, visto que esta é a 
ferramenta pela qual o farmacêutico auxilia 0 paciente nas necessidades relacionadas ao medicamento, como a solução de dúvidas sobre as doses e horários para administração do medicamento prescrito, as possíveis interações com os alimentos e/ou medicamentos e a detecção, prevenção e resolução de problemas ${ }^{(3)}$.

A característica principal do AFT é a documentação sistemática para solução dos PRMs. Essa documentação é feita, com frequência, de forma não automatizada, com o preenchimento de fichas manuais, contendo as características e funções de um prontuário do paciente, e catalogadas em $\operatorname{arquivos}^{(4)}$.

Com a popularização dos computadores, surgiram os sistemas informatizados, que promoveram a melhoria da comunicação entre os profissionais, facilitaram a recuperação das informações sobre o paciente, entre outros aspectos ${ }^{(3)}$. Além disso, os sistemas de informação estão sendo mais amplamente usados no apoio à saúde da população e nas atividades de saúde pública relacionados à prevenção e promoção de saúde, controle de doenças, vigilância e monitoramento ${ }^{(5)}$.

Pesquisas recentes sobre a avaliação da eficácia de ferramentas

\section{MATERIAL E MÉTODOS}

Embora haja variações para a condução dos métodos para o eletrônicas ou sistemas informatizados por apoiar à decisão farmacoterapêutica (que muitas vezes incluem verificação de interação medicamentosa), mostram que desempenho profissional e as taxas de erro de medicação podem melhorar com esses recursos, embora não exista nenhuma evidência de melhoria dos resultados dos pacientes ${ }^{(6)}$.

Apesar das vantagens sobre os métodos não informatizados, esses sistemas ainda apresentam problemas (como a baixa adesão dos usuários, a dificuldade de acesso durante a utilização do sistema e o custo de aquisição do produto). Alguns desses problemas podem ocorrer devido a pouca participação do usuário final, durante a etapa de especificação do produto $^{(3)}$.

Diante do exposto, foram avaliados os estudos disponíveis sobre as vantagens dos sistemas informatizados para prática da atenção farmacêutica nos últimos cinco anos. No presente estudo, pode ser utilizado como método a revisão integrativa da literatura, a qual tem como finalidade reunir e sintetizar o conhecimento científico já produzido sobre o tema investigado permitindo buscar, avaliar e sintetizar as evidências disponíveis para a sua incorporação na prática.

desenvolvimento de revisões integrativas, existem padrões a serem 
seguidos. $\mathrm{Na}$ operacionalização dessa revisão, foram utilizadas seis etapas: seleção de hipótese ou questão norteadora para a revisão; seleção dos estudos que irão compor a amostra; definição das características dos estudos; análise e interpretação dos resultados; e, relato da revisão(7).

A questão norteadora para a elaboração da presente revisão integrativa consistiu em: Quais as vantagens dos sistemas desenvolvidos para prática informatizada da atenção farmacêutica e as ferramentas utilizadas por estes, no âmbito do bom acompanhamento farmacoterapêutico dos usuários de medicamentos?

Para o levantamento dos artigos na literatura, foi realizada uma busca nas seguintes bases de dados: Literatura Latino-Americana e do Caribe em Ciências da Saúde (LILACS); Medical Literature Analysis and Retrieval Sistem on-line/National Library of Medicine MEDLINE/PUBMED e Scientific Eletronic Library Online SCIELO.

Foram utilizados, para busca dos artigos, os seguintes descritores e suas combinações nas línguas portuguesa, inglesa e espanhola: Sistemas informatizados (information system, eprescribing system, software e sistema de información), Atenção farmacêutica (pharmaceutical care e atención farmacéutica), Farmacovigilância (pharmacovigilance e farmacovigilancia) e Acompanhamento farmacoterapêutico (follow-up pharmacoterapy e segumiento farmacoterapeutico).

Os critérios de inclusão definidos para a seleção dos artigos foram: artigos publicados em português, inglês ou espanhol; artigos na íntegra que retratassem a temática referente à revisão integrativa e artigos originais publicados $e$ indexados nos referidos bancos de dados com livre acesso e publicados nos últimos cinco anos.

Já os critérios de exclusão consistiram em série de casos, relatos de casos informais, capítulos de livros, teses, dissertações, trabalhos de conclusão de curso, reportagens, notícias, editoriais, textos não científicos e artigos científicos de revisão sem disponibilidade do texto na íntegra Online ou em outro idioma diferente dos adotados nos critérios de inclusão. Além disso, foi adotado como critério de inclusão os artigos repetidos em mais de uma base de dados e aqueles que não ressaltavam as vantagens/desvantagens quanto ao uso de sistemas informatizados na prática da atenção farmacêutica.

O instrumento utilizados para extrair os dados dos estudos foi um formulário semelhante aos dados descritos nas tabelas 1 e 2 , extraindo as informações quanto título, autoria, objetivos e resultados/conclusão.

Dos 69 artigos encontrados após a busca dos estudos e obedecendo rigorosamente aos critérios de inclusão 
FREITAS, M. R.

REF - ISSN 1808-0804 Vol. XI (1), 35 - 47, 2014.

apresentados, foi realizada a leitura do título e do resumo de cada artigo científico, com o objetivo de verificar a sua adequação com a pergunta norteadora da presente revisão integrativa.

A partir de então, dos 69 artigos encontrados foram incluídos apenas 15

\section{RESULTADOS}

$\mathrm{Na}$ presente revisão integrativa, foram analisados 15 artigos científicos que atenderam rigorosamente à seleção da amostra previamente estabelecida e, após a leitura e análise dos mesmos, foram apresentados nas Tabelas 1A, 1B artigos dos quais estavam presentes um artigo na base de dados LILACS, doze no MEDLINE, somente um artigo científico no PUBMED e um na base de dados SCIELO. Os 54 artigos que não atendiam aos critérios de inclusão foram descartados da revisão integrativa. e 2 de acordo com a síntese desses artigos científicos selecionados, segundo título, autoria, objetivos e resultados/conclusão nas quatro bases de dados: PUBMED, SCIELO, MEDLINE e LILACS.

Tabela 1A: Síntese dos estudos descritivos selecionados para revisão.

\begin{tabular}{|c|c|c|c|}
\hline TÍTULO & AUTORES & OBJETIVOS & RESULTADOS / CONCLUSÃO \\
\hline $\begin{array}{l}\text { I - Aplicación de la tecnología } \\
\text { web } 2.0 \text { en un centro de } \\
\text { información } \\
\text { de medicamento }\end{array}$ & $\begin{array}{c}\text { Giménez et } \\
\mathrm{al}^{(8)}\end{array}$ & $\begin{array}{l}\text { Desenvolver um portal Web } 2.0 \\
\text { e selecionar dados úteis em um } \\
\text { Centro de Informação sobre } \\
\text { medicamentos em Farmácia } \\
\text { Hospitalar }\end{array}$ & $\begin{array}{l}\text { Através do portal criado, dados } \\
\text { foram usados por } 5 \text { meses para } \\
\text { gerar a informação para avaliação } \\
\text { da equipe médica e fornecer } \\
\text { visibilidade externa para outros } \\
\text { profissionais de saúde }\end{array}$ \\
\hline $\begin{array}{l}\text { II - Construction of a Multisite } \\
\text { Data Link Using Electronic } \\
\text { Health Records for the } \\
\text { Identification, Surveillance, } \\
\text { Prevention, and Management } \\
\text { of Diabetes Mellitus: The } \\
\text { SUPREME-DM Project }\end{array}$ & Nichols et $\mathrm{al}^{(9)}$ & $\begin{array}{l}\text { Identificar o número de } \\
\text { pessoas com diabetes a partir } \\
\text { de um Data Link diabetes } \\
\text { desenvolvido como parte do } \\
\text { projeto SUPREMO-DM } \\
\text { (vigilância, prevenção e } \\
\text { controle do diabetes Mellitus) }\end{array}$ & $\begin{array}{l}\text { Os } 11 \text { sistemas de saúde } \\
\text { encontraram } 6,9 \% \text { de pacientes que } \\
\text { preencheram um ou mais critérios do } \\
\text { estudo para a diabetes, o que } \\
\text { melhorou o acompanhamento } \\
\text { farmacoterapêutico desses usuários }\end{array}$ \\
\hline $\begin{array}{l}\text { III - Desarrollo de un } \\
\text { buscador de información } \\
\text { farmacoterapéutica no } \\
\text { publicada en revistas } \\
\text { biomédicas }\end{array}$ & $\begin{array}{l}\text { Pazo-Oubina } \\
\text { et } \mathrm{al}^{(10)}\end{array}$ & $\begin{array}{c}\text { Identificar produtores de } \\
\text { informação farmacoterapêutica } \\
\text { não publicados em revistas } \\
\text { e desenvolver um sistema de } \\
\text { acesso }\end{array}$ & $\begin{array}{l}\text { Desenvolveu-se um navegador onde } \\
\text { é possível localizar informações } \\
\text { farmacoterapêuticas importantes } \\
\text { para o tratamento medicamentoso }\end{array}$ \\
\hline $\begin{array}{l}\text { IV - Meaningful use of } \\
\text { electronic prescribing in } 5 \\
\text { exemplar primary care } \\
\text { practices }\end{array}$ & $\begin{array}{l}\text { Crosson et } \\
\mathrm{al}^{(11)}\end{array}$ & $\begin{array}{l}\text { Identificar a aplicação } \\
\text { técnicas de utilização } \\
\text { prescrições eletrônicas }\end{array}$ & $\begin{array}{l}\text { Muitas das estratégias associadas à } \\
\text { implantação bem sucedida de e- } \\
\text { prescrição foram importantes em } \\
\text { várias fases, possibilitando o melhor } \\
\text { acesso farmacêutico }\end{array}$ \\
\hline $\begin{array}{l}\mathbf{v} \text { - Prescriber and staff } \\
\text { perceptions of an electronic } \\
\text { prescribing system in primary } \\
\text { care: a qualitative assessment }\end{array}$ & Devine et $\mathrm{al}^{(12)}$ & $\begin{array}{l}\text { Descrever percepções de } \\
\text { implementação de um sistema } \\
\text { de prescrição eletrônica no } \\
\text { ambulatório }\end{array}$ & $\begin{array}{l}\text { Maior disponibilidade } \\
\text { de informação clínica de sistemas } \\
\text { informatizados resultou na prescrição } \\
\text { eficaz e de maior cuidado }\end{array}$ \\
\hline
\end{tabular}


FREITAS, M. R.

REF - ISSN 1808-0804 Vol. XI (1), 35 - 47, 2014.

Tabela 1B: Síntese dos estudos descritivos selecionados para revisão.

\begin{tabular}{|c|c|c|c|}
\hline TÍTULO & AUTORES & OBJETIVOS & RESULTADOS / CONCLUSÃO \\
\hline $\begin{array}{l}\text { VI - An electronic health } \\
\text { record-enabled obesity } \\
\text { database }\end{array}$ & Wood et $\mathrm{al}^{(13)}$ & $\begin{array}{l}\text { Desenvolver sistema para a } \\
\text { obtenção de dados de } \\
\text { pacientes submetidos a cirurgia } \\
\text { gástrica }\end{array}$ & $\begin{array}{l}\text { Mais de } 74 \% \text { dos pacientes tinham } \\
\text { dados pós-operatórios de peso } \\
\text { disponíveis em } 4 \text { anos }\end{array}$ \\
\hline $\begin{array}{l}\text { VII - The Influence that } \\
\text { electronic prescribing has on } \\
\text { medication } \\
\text { errors and preventable adverse } \\
\text { drug events: an interrupted } \\
\text { time-series study }\end{array}$ & $\begin{array}{c}\text { Van Doormaal } \\
\text { et al }\end{array}$ & $\begin{array}{l}\text { Avaliar o efeito de um sistema } \\
\text { informatizado de prescrição } \\
\text { médica eletrônica sobre a } \\
\text { incidência de erros de } \\
\text { medicação evitar } \\
\text { eventos adversos }\end{array}$ & $\begin{array}{l}\text { A pós-implementação do sistema } \\
\text { provocou redução de prescrições com } \\
\text { erros de medicação, porém não } \\
\text { exatamente reações adversas }\end{array}$ \\
\hline $\begin{array}{l}\text { VIII - Using Primary Care } \\
\text { Prescribing Databases For } \\
\text { Pharmacovigilance }\end{array}$ & $\begin{array}{c}\text { Mohamed et } \\
\mathrm{al}^{(15)}\end{array}$ & $\begin{array}{l}\text { Desenvolver um sistema de } \\
\text { monitoramento prm capaz de } \\
\text { identificar as razões para a } \\
\text { descontinuação do tratamento } \\
\text { prescrito }\end{array}$ & $\begin{array}{l}\text { Através do sistema desenvolvido } \\
\text { foram identificados que } 19,4 \% \\
(193) \text { dos pacientes que } \\
\text { interromperam o medicamento tinha } \\
\text { um PRM registado na base de dados }\end{array}$ \\
\hline $\begin{array}{l}\text { IX - Farmacovigilância: um } \\
\text { passo em direção ao uso } \\
\text { racional de plantas medicinais } \\
\text { e fitoterápicos }\end{array}$ & $\begin{array}{c}\text { Balbino et } \\
\text { al }^{(16)}\end{array}$ & $\begin{array}{l}\text { Avaliação das notificações de } \\
\text { eventos adversos a plantas } \\
\text { medicinais e fitoterápicos } \\
\text { efetuadas voluntariamente ao } \\
\text { Sistema Nacional de } \\
\text { Farmacovigilância no período } \\
\text { de janeiro de } 1999 \text { a março de } \\
2009 \text {. }\end{array}$ & $\begin{array}{l}\text { Aplicando-se o critério de inclusão, } \\
71 \text { notificações de eventos adversos } \\
\text { tiveram plantas medicinais ou } \\
\text { fitoterápicos como principal suspeito. }\end{array}$ \\
\hline $\begin{array}{l}\text { X - Proposta de Sistema de } \\
\text { Informação para Atenção } \\
\text { Farmacêutica baseado no } \\
\text { Método Dáder }\end{array}$ & $\begin{array}{c}\text { Yokoyama et } \\
\text { al }^{(17)}\end{array}$ & $\begin{array}{l}\text { O desenvolvimento de um } \\
\text { sistema baseado no método } \\
\text { Dáder, facilitando r o } \\
\text { acompanhamento } \\
\text { farmacoterapêutico. }\end{array}$ & $\begin{array}{l}\text { O sistema está em fase de } \\
\text { atualização constante, buscando } \\
\text { incluir os dados de cada usuário }\end{array}$ \\
\hline
\end{tabular}

Tabela 2: Síntese dos artigos experimentais selecionados para a revisão

\begin{tabular}{|c|c|c|c|}
\hline TITULO & AUTORES & OBJETIVOS & RESULTADOS/CONCLUSÃO \\
\hline $\begin{array}{c}\text { XI - Electronic prescribing } \\
\text { improves medication safety in } \\
\text { community-based office } \\
\text { practices }\end{array}$ & $\begin{array}{c}\text { Kaushal et } \\
\text { al }^{(18)}\end{array}$ & $\begin{array}{l}\text { Avaliar o impacto de um sistema de e- } \\
\text { prescrição sobre as taxas de erros de } \\
\text { prescrição num ambulatório }\end{array}$ & $\begin{array}{c}\text { Em um ano, a taxa de erro para } \\
\text { e-prescrição foi } \\
\text { significativamente menor do que } \\
\text { para não adeptos. }\end{array}$ \\
\hline $\begin{array}{l}\text { XII - Evaluating user } \\
\text { satisfaction with an electronic } \\
\text { prescription system in a } \\
\text { primary care group }\end{array}$ & Tan et $\mathrm{al}^{(19)}$ & $\begin{array}{l}\text { Analisar a satisfação dos usuários com } \\
\text { um sistema de prescrição eletrônica } \\
\text { implementado no Grupo Policlínicas } \\
\text { em Singapura }\end{array}$ & $\begin{array}{l}\text { Médicos relataram que a } \\
\text { prescrição eletrônica reduz erros } \\
\text { de prescrição e intervenções }\end{array}$ \\
\hline $\begin{array}{l}\text { XIII - The effects of electronic } \\
\text { prescribing on the quality of } \\
\text { prescribing }\end{array}$ & $\begin{array}{c}\text { Donyai et } \\
\text { al }^{(20)}\end{array}$ & $\begin{array}{l}\text { Investigar os efeitos da prescrição } \\
\text { electrónica (PE) sobre a prescrição } \\
\text { tal como indicado por erros de } \\
\text { prescrição em um hospital do Reino } \\
\text { Unido. }\end{array}$ & $\begin{array}{l}\text { Na sequência da introdução de } \\
\text { EP, houve uma redução } \\
\text { significativa nas intervenções } \\
\text { farmacêuticas e nos erros de } \\
\text { prescrição. }\end{array}$ \\
\hline $\begin{array}{l}\text { XIV - Computerized clinical } \\
\text { decision support during } \\
\text { medication ordering for long- } \\
\text { term care residents with renal } \\
\text { insufficiency }\end{array}$ & Field et $\mathrm{al}^{(6)}$ & 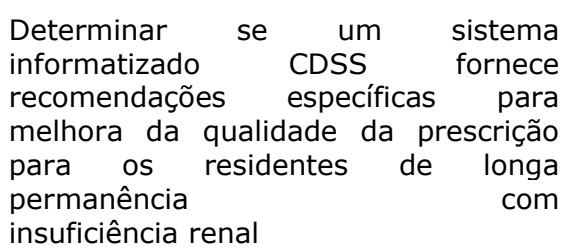 & $\begin{array}{c}\text { Recomendações através de } \\
\text { CDSSs foram mais fidedignas na } \\
\text { intervenção no âmbito do risco } \\
\text { das drogas utilizadas }\end{array}$ \\
\hline
\end{tabular}




$\begin{array}{cclcc}\mathbf{X V} \text { - Perceptions of Standards- } & \text { Wang et } & \text { Comparar as experiências de } & \text { E-prescritores eram mais } \\ \text { based Electronic Prescribing } & \text { al }^{(22)} & \begin{array}{c}\text { prescrição de usuários e não usuários } \\ \text { de sistemas de e-prescrição }\end{array} & \begin{array}{c}\text { aceitáveis do que não-e- } \\ \text { prescritores }\end{array} \\ \begin{array}{c}\text { as Implemented in Outpatient } \\ \text { Primary Care: A Physician }\end{array} & & & \\ \text { Survey } & & & \end{array}$

Os quinze artigos selecionados estavam distribuídos entre os seguintes periódicos: Journal of the American Medical Informatics Association (três artigos), BMC Medical Informatics and Decision Making (dois artigos), Annals Academy of Medicine (dois artigos), Farmácia Hospitalaria (dois artigos), British Journal of Clinical Pharmacology (dois artigos), The Journal of General Internal Medicine (um artigo), Brazilian Journal of Pharmacognosy (um artigo), Revista de Ciências Farmacêuticas Básica e Aplicada (um artigo) e PDC Preventing Chronic Disease (um artigo) os quais estão apresentados por país na Figura 1.

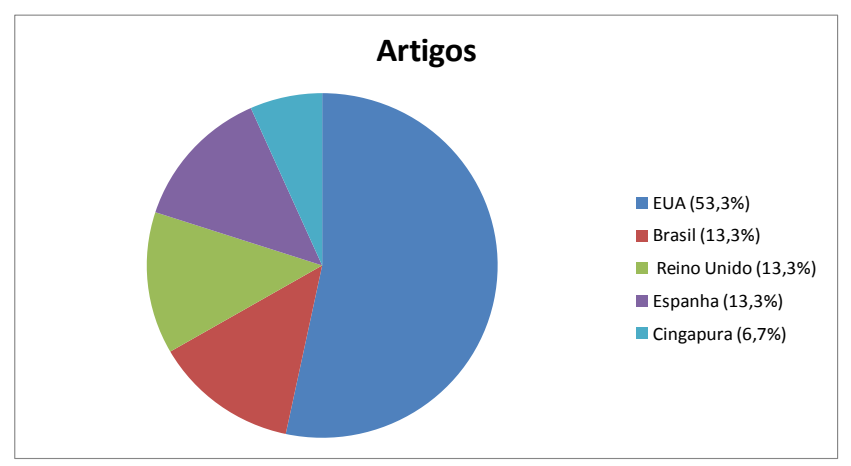

Figura 1: Distribuição amostral dos artigos científicos quanto aos países nos quais foram publicados.

Quanto aos objetivos propostos pelas publicações foram identificados que a maioria abordava conceitos de tecnologia da informação, problemas relacionados a medicamento, métodos

\section{DISCUSSÃO}

\section{A importância de prática}

\section{baseada em evidências científicas}

A Prática Baseada em Evidências (PBE) é um processo de descoberta, de prescrição, tratamento, intervenções farmacêuticas e a relação entre uso de sistemas informatizados e o acompanhamento farmacoterapêutico.

avaliação e aplicação de evidências científicas para $o$ tratamento e gerenciamento da saúde ${ }^{(7)}$. Assim, os estudos de atenção farmacêutica que adotam o método de pesquisa integrativa agregam conhecimentos previamente construídos e podem 
colaborar para consolidar saberes e oferecer elementos para a elaboração de intervenções farmacoterapêuticas. Além disso, podem favorecer a incorporação e a aplicabilidade de práticas ainda pouco empreendidas. A atenção farmacêutica baseada em evidências enfatiza o uso de pesquisas para guiar as tomadas de decisão clínica e pode ser definida como o uso consciente, explícito e criterioso de informações derivadas de pesquisas para a intervenção eficaz na terapia medicamentosa.

Sistemas informatizados como

ferramenta para atenção
farmacêutica

Com o desenvolvimento da atenção farmacêutica e do AFT, surgiu a necessidade da documentação criteriosa, sistemática e cronológica do paciente de forma a guardar seus dados clínicos e isto gera, com frequência, fichas e arquivos escritos manualmente utilizando um grande volume de papel. A partir desse fato, foram, então, desenvolvidos os sistemas informatizados que, geralmente, possuem as características do prontuário do paciente ${ }^{(3)}$.

O Prontuário Eletrônico do Paciente (PEP) é a versão informatizada do prontuário em papel do paciente, e tem como proposta unir todos os diferentes tipos de dados de um paciente. O PEP é um sistema especificamente desenhado para apoiar os usuários, fornecendo acesso a um completo conjunto de dados, sistemas de avisos e alertas, sistemas de apoio à decisão e outros recursos que agreguem valor ao sistema e favorecem o monitoramento dos usuários. Ele apresenta vantagens relevantes em relação ao prontuário em papel: (1). Compartilhamento simultâneo e remoto de dados; (2). Dados sempre legíveis; (3). Oferecimento de apoio ao diagnóstico e à tomada de decisão; (4). Possibilidade de variação na forma de visualizar os dados; (5). Maior possibilidade de segurança quanto à perda de dados devido à possibilidade de backups; (6). Maior possibilidade de segurança quanto à confidencialidade dos dados devido à possibilidade de controles eletrônicos de acesso; (7). O sistema pode consistir e não permitir procedimentos que não constem nos protocolos envolvidos entre outras $^{(22)}$.

Logo, com o auxílio de tecnologias de Inteligência Artificial aos bancos de dados clínicos, os farmacêuticos e outros profissionais da saúde podem utilizá-los para tomar decisões baseadas na situação particular de um paciente e intervenções por exemplo, para selecionar o melhor antibiótico economizando custos em medicamentos e, ao mesmo tempo, diminuindo a mortalidade ${ }^{(5)}$.

A proposta de um sistema informatizado transmitida ao usuário de 
forma clara e fácil é um desafio contínuo, sendo necessários constantes testes e pesquisas sobre o comportamento do usuário a fim de entender diferenças de perfis, limitações, formas de utilização e outros. São evidentes as expectativas do usuário quanto à necessidade de sistemas ágeis, fáceis e que consumam pouco tempo de operação(22). O uso desses softwares na atenção farmacêutica disponibiliza um melhor acompanhamento farmacoterapêutico dos usuários de medicamentos além de uma maior segurança aos mesmos e também aos farmacêuticos responsáveis ${ }^{(9)}$

Os artigos I, III e X da tabela 1 exemplificam 0 desenvolvimento de sistemas simples e práticos de atenção à saúde a partir da engenharia de software. O Centers for Disease Control and Prevention (CDC), demonstra que a partir da criação de sites como o descrito no artigo II, é possível a padronização de dados dos pacientes, conduzindo a um tratamento mais eficaz e maior nível de monitoramento de portadores de doenças crônicas $^{(9)}$. O artigo IX descreve ainda o uso de softwares no âmbito da farmacovigilância de medicamentos, como os fitoterápicos, a fim de possibilitar disponibilização de informações no sítio eletrônico da Anvisa (Agência Nacional de Vigilância Sanitária) e, quando necessário, inclusão de novos eventos adversos nas bulas dos medicamentos, publicação de alertas e inspeções em empresas fabricantes.

Todas as publicações descritas na Tabela 2 enfatizam as vantagens da tecnologia da informação e os sistemas informatizados durante 0 acompanhamento farmacoterapêutico, de modo que as experiências realizadas, seja em ambiente hospitalar (artigos XIII, XII) ou ambulatorial (artigos XI), demonstraram resultados benéficos.

Os usuários dos sistemas de prescrição eletrônica revelaram que seu uso implica em um maior acesso a informações que ajudam na redução de interações (medicamento/medicamento e/ou medicamento/alimento) e de intervenções farmacêuticas, como aquelas realizadas por chamadas telefônicas e, portanto, aumentam a eficácia do tratamento medicamentoso ${ }^{(21)}$.

No entanto, apesar desses aspectos positivos quanto à tecnologia empregada na atenção farmacêutica, segundo os autores do artigo XII, reduções nos erros de prescrição ou intervenções farmacêuticas que são facilitados por uma melhor legibilidade dos sistemas de prescrições eletrônicas; implementação de alertas de interação medicamentosa, hipersensibilidades e outras utilidades dos sistemas de computadores não estão diretamente associados com os níveis de satisfação destes. A falta de habilidades quanto a 
operação desses sistemas dificultam a implementação dos mesmos.

A introdução de sistemas de prescrição eletrônica como apoio à decisão clínica (CDSS) gera uma grande redução em erros de prescrição $^{(6)}$. Porém, esta redução substancial de erros não foi seguida de uma redução significativa na incidência de reações adversas $^{(14)}$. Uma das razões apresentadas é a utilização de CDSS pouco precisos. O artigo VIII demonstra que a partir da implantação de sistemas modernos capazes de armazenar dados mais detalhados do paciente houve significativa redução dos eventos adversos e, consequente maior adesão ao tratamento medicamentoso.

Além disso, nem sempre o usuário terá conhecimento das necessidades de segurança, principalmente técnicas de sistemas informatizados em atenção à saúde. E isso pode causar conflitos entre o desejo do usuário e os controles de segurança. Assim, um especialista de engenharia de software deverá mapear tanto os requisitos funcionais quanto os de segurança, e especialistas em segurança da informação deverão escolher os controles técnicos $\operatorname{adequados}^{(17)}$. Logo, a validação desses sistemas deve ser certificada ${ }^{(23)}$ e a privacidade e a confidencialidade dos pacientes devem ser protegidas, resguardando o acesso a essas fontes de informação, incluindo os bancos de dados informatizados e suas cópias de segurança(17).

\section{Certificação e validação de sistemas informatizados em saúde}

Devido à sua importância e à possibilidade de erros afetarem 0 paciente, na maioria dos países, alguns sistemas de informação em saúde como os softwares de PEP têm de passar por um processo de certificação de qualidade e aderência a princípios básicos de segurança e confidencialidade ${ }^{(5)}$.

No entanto, quanto ao uso de sistemas informatizados em saúde ainda são excassas as evidências científicas, principalmente em âmbito nacional (Figura 1).

No Brasil, existe um processo de certificação (desenvolvido a partir de 2002) operacionalizado pela SBIS (Sociedade Brasileira de Informática em Saúde) ${ }^{(23)}$ em parceria com o CFM (Conselho Federal de Medicina). Esse processo é destinado a Sistemas de Registro Eletrônico de Saúde. O objetivo principal dessa certificação é melhorar a qualidade dos sistemas de informação em saúde no Brasil, bem como dar um suporte técnico e jurídico, especialmente, para o uso de prontuários eletrônicos. Apenas em novembro de 2009 foi emitido o primeiro certificado, o que demonstra a carência na área(22) 0 processo de certificação é voluntário, mas muito valorizado, uma vez que pode 
ser entendido como uma "opinião técnica qualificada e imparcial" de duas instituições dispostas a garantir a privacidade e confidencialidade da

\section{CONSIDERAÇÕES FINAIS}

Os objetivos propostos foram alcançados visto que, a partir das evidências científicas analisadas foram constatadas as vantagens estabelecidas pelo uso de sistemas de informação no acompanhamento farmacoterapêutico, auxiliando os farmacêuticos a atuarem positivamente durante 0 acompanhamento farmacoterapêutico e, consequentemente, no serviço de saúde como um todo. A informatização dos dados e do processo economiza tempo e favorece a tomada da decisão clínica de forma adequada, já que é possível gerar diversos relatórios para comparar os informação de saúde, atender a legislação brasileira sobre documentos eletrônicos e melhorar a qualidade dos sistemas de informação em saúde ${ }^{(23)}$.

\begin{abstract}
parâmetros dos pacientes, sobre os PRMs detectados, as intervenções que necessitam ser realizadas, entre outros. Entretanto, a privacidade e a confidencialidade dos pacientes devem ser protegidas. Para isso, é necessário o auxílio da engenharia de software junto ao conhecimento farmacêutico na elaboração desses sistemas informatizados. Além disso, é imprescindível a implementação de cursos para treinamento dos usuários desses sistemas para uma incorporação eficaz de sistemas informatizados à prática farmacêutica.
\end{abstract}

\section{REFERÊNCIAS BIBLIOGRÁFICAS}

1. Martinez RF, Fernandes LF, Gastelurrutia MA, Parras M, Faus MJ. Programa dader de seguimiento del tratamiento farmacológico: Resultados de la fase piloto. Ars Pharm. 2001;42:53-65.

2. Novaes MRCG. Atenção farmacêutica e o uso racional de medicamentos em idosos. Pharm Bras. 2007;59:86-88.

3. Yokoyama CS. Sistema de informação para acompanhamento farmacoterapêutico em farmácia comunitária [dissertação]. Curitiba: Pontifícia Universidade Católica/PUC-PR. 2010. 141p. 
4. Machuca M, Llimós FF, Faus JM. Método Dáder manual de acompanhamento farmacoterapêutico. Grupo de Investigação em Atenção Farmacêutica (CTS-131). Granada: Universidade de Granada; 2003. 46p.

5. Pinochet LHC. Tendências de Tecnologia de Informação na Gestão da Saúde. Mundo Saúde. 2011; 35(4):382-394.

6. Field TS, Rochon P, Lee M, Gavendo L, Baril JL, Gurwitz JH. Computerized Clinical Decision Support During Medication Ordering for Long-term Care Residents with Renal Insufficiency. J Am Med Inform Assoc. 2009;16:480-485.

7. Souza MT, Silva MD, Carvalho R. Revisão integrativa: o que é como fazer. Einstein. 2010;8:102-6.

8. Giménez JCJ, González CP, Vadell CV, Lacambra MEP, Otero JV, Dapena MJC. Aplicación de la tecnología web 2.0 en un centro de información de medicamentos. Farm Hosp. 2011;35(6):315e1-315e5.

9. Nichols GA, Desay J, Lafata JE, Lawrence JM, O'Connor PJ, Pathak RD et al. Construction of a Multsite DataLink Using Eletronic Health Records for the Identification, Surveillance, Prevention, and Management of Diabetes Mellitus: The SUPREME-DM Project.PCD - Preventing Chronic Disease. 2012;9:1-9.

10. Pazo-Oubiña F, Pita CC, Latorre FP, Periañez-Parrága L, Bosch PV. Desarrollo de un buscador de información farmacoterapéutica no publicada em revistas biomédicas. Farm Hosp. 2011;35(5):254e1-254e5.

11. Crosson JC, Etz RS, Wu S, Straus SG, Eisenman D, Bell DS. Meaningful Use of Electronic prescribing in 5 Exemplar Primary Care. Ann Fam Med. 2011;9(5):392-397.

12. Devine EB, Williams EC, Martin DP, Sitting DF, Hornoch PT, Payne TH et al. Prescriber and staff perceptions of an electronic prescribing system in primary care: a qualitative assessment. BMC Med Inform Decis Mak. 2010;10:72-84.

13. Wood C, Chu X, Manney C, Strodel W, Petrik A, Gabrielsen J et al. An electronic health record-enabled obesity database. BMC Med Inform Decis Mak. 2012;12:45-53. 
14. Van Doormal JE, Bemt PMLAVD, Zaal RJ, Egberts ACG, Lenderink BW, Kosterink JGW et al. The Influence that Electronic Prescribing Has on Medication Errors and Preventable Adverse Drug Events: an Interrupted Time-series Study. J Am Med Inform Assoc. $2009 ; 16(6): 816-825$.

15. Mohamed IN, Helms PJ, Simpson CR, Milne RM, McLay JS. Using primary care prescribing databases for pharmacovigilance. Br J Clin Pharmacol. 2010;71(2):244-249.

16. Balbino EE, Dias MF. Farmacovigilância: um passo em direção ao uso racional de plantas medicinais e fitoterápicos. Braz J Pharmacog. 2010;20(6):992-1000.

17. Yokoyama CS, Malucelli A, Moro CMC, Hirano LR, Nohama P. Proposta de Sistema de Informação para Atenção Farmacêutica baseado no Método Dáder. Rev Ciênc Farm. Básica Apl. 2011;32(1):19-26.

18. Kaushal R, Kern LM, Barrón Y, Quaresimo J, Abramson EL. Electronic Prescribing Improves Medication Safety in Community-Based Office Practices. J Gen Intern Med. 2010;25(6):530-536.

19. Tan WS, Phang JSK, Tan LK. Evaluating User Satisfaction with an Electronic Prescription System in a Primary Care Group. Ann Acad Med. 2009;38:494-500.

20. Donyai P, O'Grady K, Jacklin A, Barber N, Franklin BD. The effects of electronic prescribing on the quality of prescribing. $\mathrm{Br} J$ Clin Pharmacol. 2007;65(2):230-237.

21. Wang CJ, Patel MH, Schueth AJ, Bradley M, Wu S, Crosson JC et al. Perceptions of Standards-based Electronic Prescribing Systems as Implemented in Outpatient Primary Care: A Physician Survey. J Am Med Inform Assoc. 2009;16(4):493-502.

22. Paiva PB, Pereira SR. A importância da Engenharia da Usabilidade para a Segurança de Sistemas Informatizados em Saúde. J. Health Inform. 2011;3(3):123-129.

23.SBIS. Sociedade Brasileira de Informática em Saúde. 2012. Disponível em: < http://www.sbis.org.br > Acesso em 23 out 2012.

24. Wong K, Yu SKH, Holbrook A. A systematic review of medication safety outcomes related to drug interaction software. J Popul Ther Clin Pharmaco. 2010;17(2):e243-e255. 